\title{
Pediatric cranial deformations: demographic associations
}

\author{
Jarrett Foster, BS, ${ }^{1,2}$ Ranbir Ahluwalia, BS, ${ }^{1,3}$ Madeleine Sherburn, BA, ${ }^{1}$ Katherine Kelly, BS ${ }^{1,6}$ \\ Georgina E. Sellyn, MA, ${ }^{1}$ Chelsea Kiely, ${ }^{1,4}$ Alyssa L. Wiseman, MS, ${ }^{1}$ Stephen Gannon, CCRP,1 \\ Chevis N. Shannon, DrPH, MBA, ${ }^{1,5}$ and Christopher M. Bonfield, MD ${ }^{1,5}$
}

${ }^{1}$ Surgical Outcomes Center for Kids, Monroe Carell Jr. Children's Hospital at Vanderbilt, Nashville, Tennessee; ${ }^{2}$ University of South Carolina School of Medicine, Columbia, South Carolina; ${ }^{3}$ Florida State University College of Medicine, Tallahassee, Florida; ${ }^{4}$ Department of Neurobiology, Cornell University, Ithaca, New York; ${ }^{5}$ Department of Neurological Surgery, Vanderbilt University Medical Center, Nashville, Tennessee; and ${ }^{6}$ Vanderbilt University School of Medicine, Nashville, Tennessee

OBJECTIVE No study has established a relationship between cranial deformations and demographic factors. While the connection between the Back to Sleep campaign and cranial deformation has been outlined, considerations toward cultural or anthropological differences should also be investigated.

METHODS The authors conducted a retrospective review of 1499 patients (age range 2 months to less than 19 years) who presented for possible trauma in 2018 and had a negative CT scan. The cranial vault asymmetry index (CVAI) and cranial index $(\mathrm{Cl})$ were used to evaluate potential cranial deformations. The cohort was evaluated for differences between sex, race, and ethnicity among 1) all patients and 2) patients within the clinical treatment window (2-24 months of age). Patients categorized as "other" and those for whom data were missing were excluded from analysis.

RESULTS In the CVAI cohort with available data $(n=1499$, although data were missing for each variable), $800(56.7 \%)$ of 1411 patients were male, 1024 (79\%) of 1304 patients were Caucasian, 253 (19.4\%) of 1304 patients were African American, and $127(10.3 \%)$ of 1236 patients were of Hispanic/Latin American descent. The mean CVAI values were significantly different between sex $(p<0.001)$ and race $(p<0.001)$. However, only race was associated with differences in positional posterior plagiocephaly (PPP) diagnosis $(p<0.001)$. There was no significant difference in CVAI measurements for ethnicity $(p=0.968)$. Of the 520 patients in the treatment window cohort, $307(59 \%)$ were male. Of the 421 patients with data for race, 334 were Caucasian and 80 were African American; 47 of the 483 patients with ethnicity data were of Hispanic/Latin American descent. There were no differences between mean CVAI values for sex $(p=$ $0.404)$ or ethnicity $(p=0.600)$. There were significant differences between the mean CVAI values for Caucasian and African American patients $(p<0.001)$ and rate of PPP diagnosis $(p=0.02)$. In the $\mathrm{Cl}$ cohort with available data $(n=$ 1429 , although data were missing for each variable), $849(56.8 \%)$ of 1494 patients were male, $1007(67.4 \%)$ of 1283 were Caucasian, $248(16.6 \%)$ of 1283 were African American, and 138 patients with ethnicity data $(n=1320)$ of Hispanic/Latin American descent. Within the clinical treatment window cohort with available data, $373(59.2 \%)$ of 630 patients were male, 403 were Caucasian (81.9\%), 84 were African American (17.1\%), and 55 (10.5\%) of 528 patients were of Hispanicl Latin American descent. The mean $\mathrm{Cl}$ values were not significantly different between sexes $(p=0.450)$ in either cohort. However, there were significant differences between $\mathrm{Cl}$ measurements for Caucasian and African American patients ( $p$ $<0.001)$ as well as patients of Hispanic/Latin American descent $(p<0.001)$ in both cohorts.

CONCLUSIONS The authors found no significant associations between cranial deformations and sex. However, significant differences exist between Caucasian and African American patients as well as patients with Hispanic/Latin American heritage. These findings suggest cultural or anthropological influences on defining skull deformations. Further investigation into the factors contributing to these differences should be undertaken.

https://thejns.org/doi/abs/10.3171/2020.3.PEDS2085

KEYWORDS brachycephaly; plagiocephaly; cephalic index; cranial vault asymmetry index; craniofacial

ABBREVIATIONS $\mathrm{Cl}=$ cranial index; CVAI = cranial vault asymmetry index; MCJCHV = Monroe Carell Jr. Children's Hospital at Vanderbilt; PPP = posterior positional plagiocephaly; SIDS = sudden infant death syndrome.

SUBMITTED February 4, 2020. ACCEPTED March 31, 2020.

INCLUDE WHEN CITING Published online May 29, 2020; DOI: 10.3171/2020.3.PEDS2085. 
$\mathrm{N}$ ONSYNOSTOTIC skull asymmetry in the pediatric neurosurgical setting refers to posterior positional plagiocephaly (PPP) and deformational brachycephaly. PPP is defined as unilateral occipital flattening with or without associated facial asymmetry, while brachycephaly is a symmetric flattening of the occiput. ${ }^{1}$ The development of deformities of this nature are usually linked to positioning habits. ${ }^{2}$ In an effort to reduce sudden infant death syndrome (SIDS), the American Academy of Pediatrics recommended that children sleep in the supine position. . $^{1,4}$ Thus, this major guideline shift changed the dynamics of force placed on the child's occiput during sleep, increasing positional deformities., ${ }^{4,5}$ Ultimately, skull deformity exists on a spectrum, and the exact characterization of skull shape can be plagiocephaly or brachycephaly, or both. ${ }^{6}$

Skull deformity can be measured in a sensitive manner using CT scans. The cranial vault asymmetry index (CVAI) can be used to measure the degree of plagiocephaly at the level of the superior orbital rim. ${ }^{7}$ In contrast, the cranial index (CI) is used to measure the degree of brachycephaly using the most lateral points of the skull and the maximum anteroposterior distance. ${ }^{8}$ These measurements have also been used to assess the development of the pediatric skull over time. ${ }^{8}$

The CI may have additional utility to determine ethnic and sex differences in skull deformity. Ultimately, the definition of a symmetric skull is an arbitrary concept. For example, the cutoff values for dolichocephaly, mesocephaly, brachycephaly, and hyperbrachycephaly vary in the current literature. ${ }^{8,9}$ Thus, skull deformity may potentially be attributed to racial and geographic variation. ${ }^{9}$ For example, Japanese and Caucasian CIs have shown significant variation in the literature. ${ }^{9}$ Thus, the purpose of our study was to determine if skull deformity can be attributed to ethnic and sex differences by using skull measurement indexes.

\section{Methods}

\section{Patient Data Collection}

After IRB approval, a cross-sectional study was con- ducted of 2262 pediatric patients evaluated for head trauma with a negative CT at Monroe Carell Jr. Children's Hospital at Vanderbilt (MCJCHV) between January 1, 2018, and December 31, 2018. The inclusion of study participants was based on the following criteria: 1) patient age $\leq 19$ years at the time of CT imaging; 2) patient evaluation at MCJCHV; and 3) CT imaging results negative for posttraumatic focal or diffuse lesions at initial presentation. Exclusion criteria were 1) history of shunted hydrocephalus; 2) radiographic presence of cranial trauma; 3) history of craniosynostosis or skull surgery; and 4) imaging complications that limited obtaining measurements. After removal for exclusion criteria, 1499 patients met the inclusion criteria and were able to be evaluated for brachycephaly via cranial index measurements, and 1429 patients met the criteria to be evaluated for plagiocephaly via CVAI measurements. Due to subtle differences in obtaining CI and CVAI, not all patients could be evaluated for both. Therefore, while the majority of the records overlap, not all of them do.

\section{PPP Evaluation}

Patient data were retrospectively reviewed through electronic medical records at MCJCHV. The CVAI was assessed using CT imaging at the level of the superior orbital rim (Figs. 1 and 2). A measurement that resulted in a $\mathrm{CVAI} \geq 3.50$ was considered to be indicative of asym-

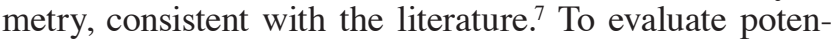
tial disparities, we evaluated our cohort for differences between sex, race, and ethnicity among 1) all patients and 2) patients within the clinical treatment window (age 2-24 months). Patients categorized as "other" and those for whom data were missing were excluded from analysis.

\section{Brachycephaly Evaluation}

The CI was calculated by measuring the cephalic width as the lateral-most point of the parietal bone and the cephalic length as the glabella to opisthocranion (Figs. 3 and 4). For CI measurements, literature has suggested that

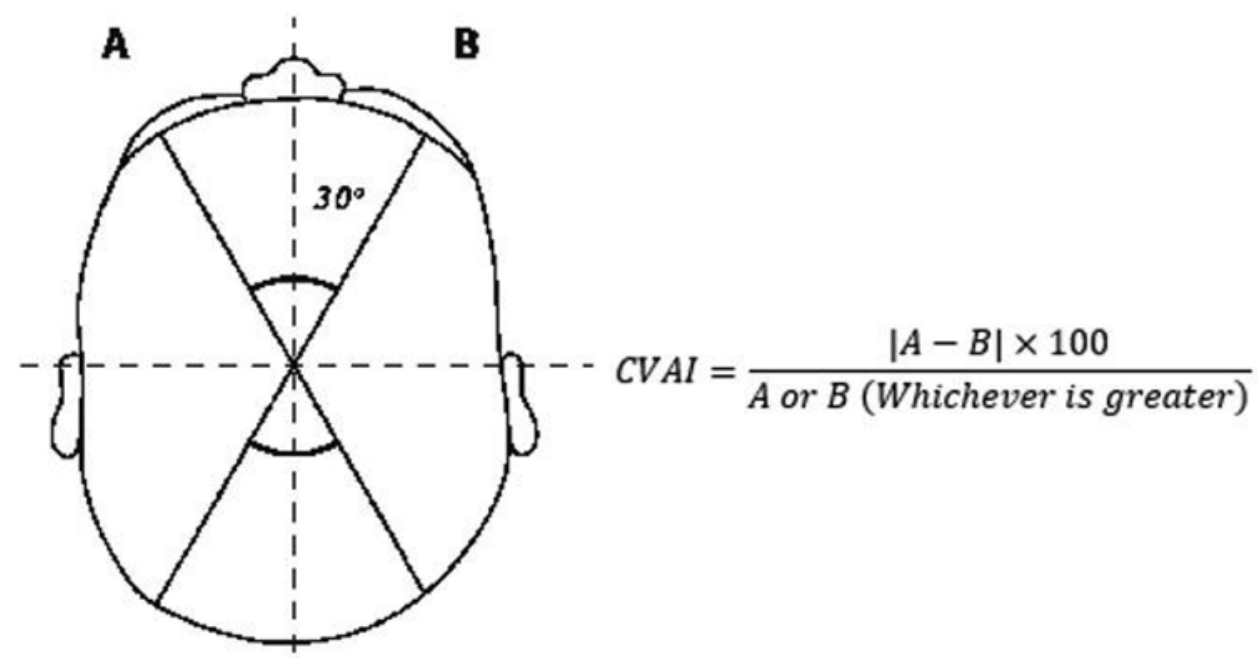

FIG. 1. Calculation of the CVAI. 

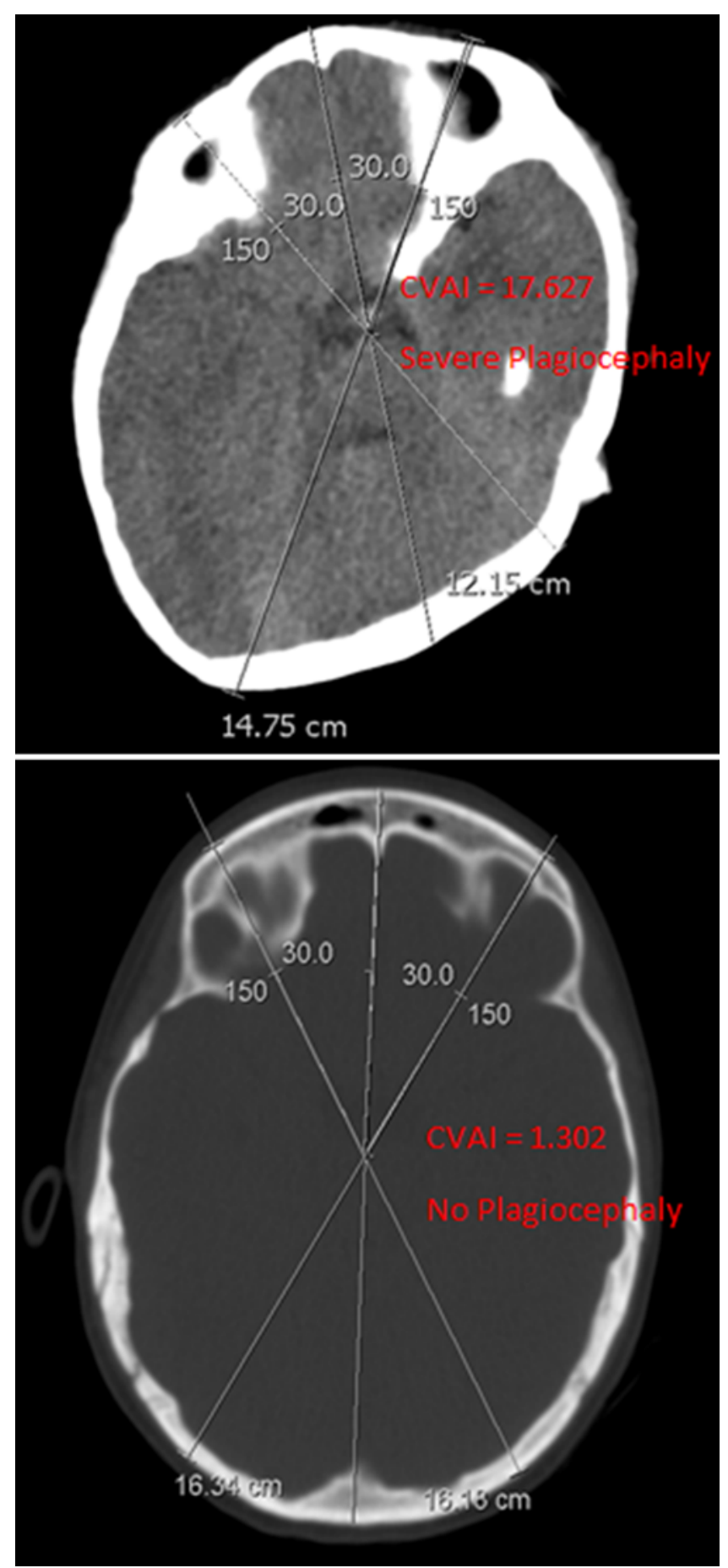

FIG. 2. Radiographic representation of CVAI measurement for brachycephaly evaluation. Figure is available in color online only.

an index greater than 81.0-95.0 is indicative of brachycephaly. ${ }^{8,10}$ To evaluate potential disparities, we evaluated our cohort for differences between sex, race, and ethnicity among 1) all patients and 2) patients within the clinical treatment window (age 2-24 months). Patients categorized as "other" and those for whom data were missing were excluded from analysis.

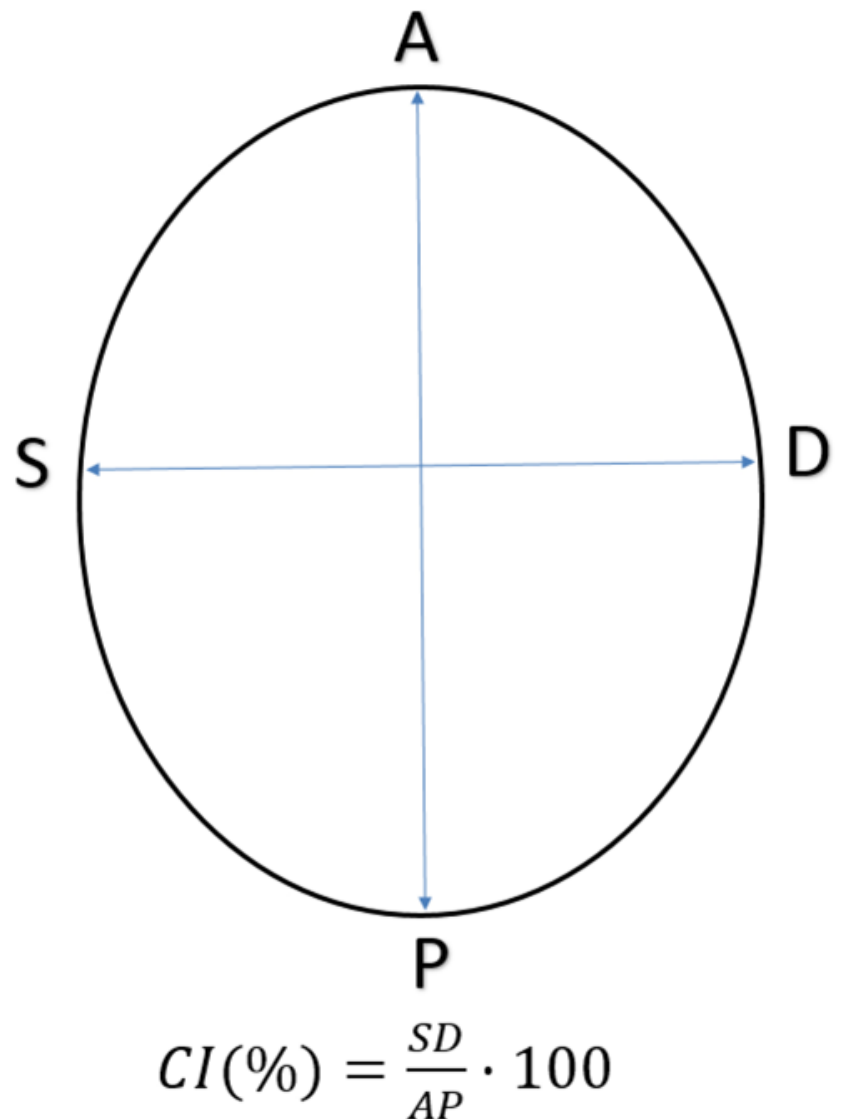

FIG. 3. Schematic representation of the $\mathrm{Cl}$ measurement. $\mathrm{A}=$ anterior; $\mathrm{D}=$ right; $\mathrm{P}=$ posterior; $\mathrm{S}=$ left. Figure is available in color online only.

\section{Statistical Analysis}

Statistical analysis was conducted using IBM SPSS (version 23, IBM Corp.). Determination of differences in CVAI mean values and their relationships to sex, race, and ethnicity were conducted using Pearson chi-square and independent t-tests. The same analysis was conducted within the subset treatment window age groups. All tests were conducted with a priori $\alpha=0.05$.

\section{Results}

The CVAI was collected for patients in the CVAI cohort to obtain plagiocephaly measurements $(n=1499$, although data were missing for each variable). Table $1 \mathrm{com}-$ pares the entire cohort with available data by sex, race, and ethnicity. A significant difference was found between males and females in terms of mean CVAI ( $p<0.001)$. Significance was also found for the mean CVAI for race ( $p<0.001)$. Specifically, a direct comparison of the differences between Caucasian and African American patients revealed significant differences in mean CVAI values $(\mathrm{p}<$ $0.001)$. However, this significance was not seen when classifying the population by ethnicity. Subsequently, patients' electronic medical records were reviewed for diagnoses of PPP. Analysis revealed no differences in diagnosis rates of PPP between sexes (64 [8.0\%] males and 33 [5.4\%] females; $\chi^{2}=3.655, p=0.056$ ). However, differences in rates of diagnosis between racial subgroups were statistically 

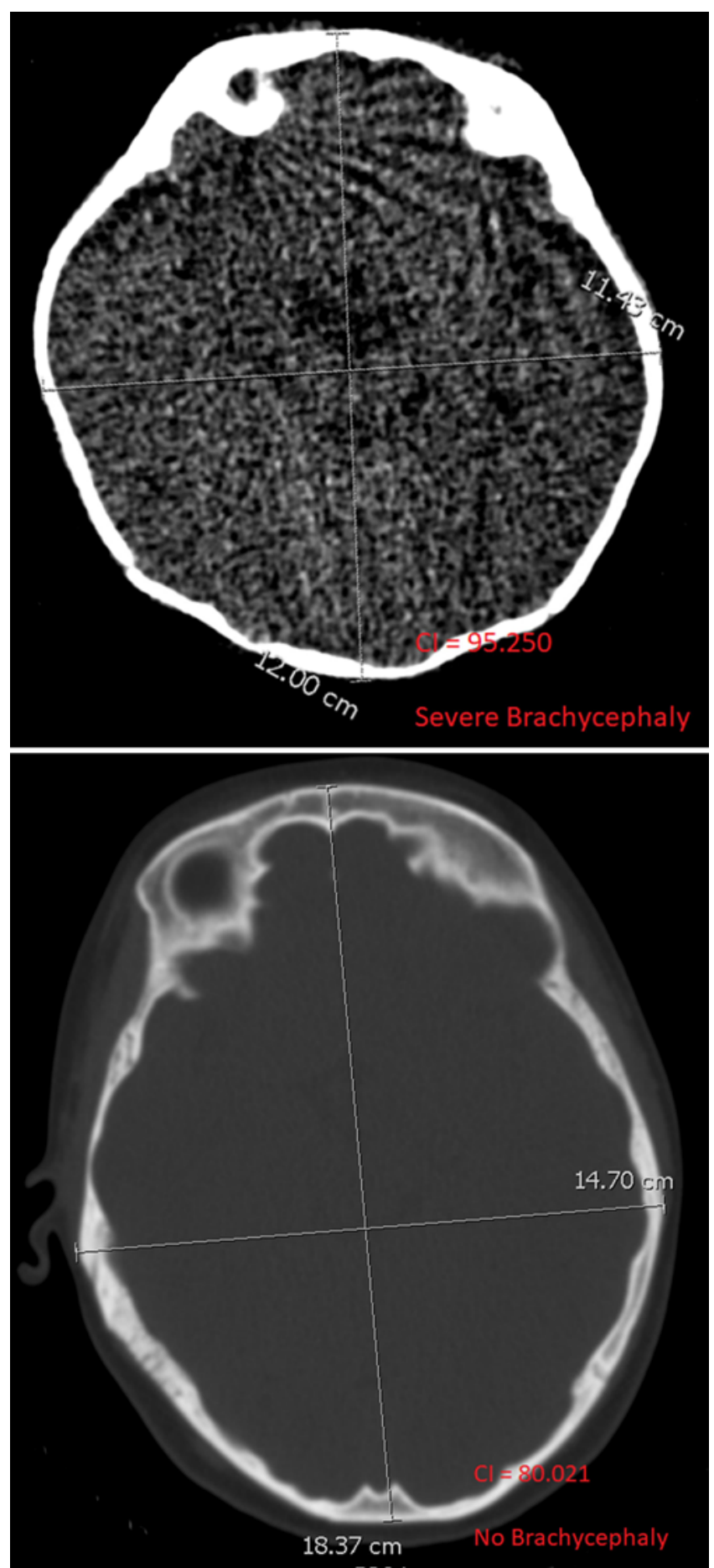

FIG. 4. Radiographic representation of the $\mathrm{Cl}$ measurement. Figure is available in color online only.

significant (65 [6.3\%] Caucasians and 6 [2.3\%] African Americans; $\chi^{2}=6.108, \mathrm{p}=0.014$ ).

A subset analysis was conducted for children who were within the treatment window of less than 24 months. CVAI was not significantly different between males and females $(\mathrm{p}=0.404)$. Additionally, mean CVAI differences were not different when the population was broken down by ethnic-
TABLE 1. Plagiocephaly demographic information for total cohort

\begin{tabular}{lccc}
\hline \multicolumn{1}{c}{ Total Cohort } & No. of Patients (\%) & Mean CVAI & p Value \\
\hline Total & 1429 & 2.574 & \\
\hline Sex $(n=1411)$ & & & $<0.001$ \\
$\quad$ Male & $800(56.7)$ & 2.718 & \\
$\quad$ Female & $611(43.3)$ & 2.384 & \\
\hline Race $(n=1304)$ & & & $<0.001^{*}$ \\
$\quad$ Caucasian & $1024(79.0)$ & 2.686 & \\
$\quad$ African American & $253(19.4)$ & 1.906 & \\
$\quad$ Asian & $22(1.7)$ & 3.368 & \\
$\quad$ American Indian & $5(0.4)$ & 3.538 & \\
\hline Ethnicity ( $n=1236)$ & & & 0.968 \\
$\quad$ Hispanic & $127(10.3)$ & 2.773 & \\
$\quad$ Non-Hispanic/Latino & $1109(89.7)$ & 2.590 & \\
\hline
\end{tabular}

Boldface type indicates statistical significance.

* Independent t-test between Caucasian and African American subgroups.

ity. However, significant differences in mean CVAI could be appreciated when the treatment cohort was stratified by race $(\mathrm{p}<0.001)$. Significant differences were also found when directly comparing Caucasian and African American patients $(\mathrm{p}<0.001)$. The subset comparison of mean CVAI based on sex, race, and ethnicity is summarized in Table 2. Analysis of this subset revealed no differences in diagnosis rates of PPP between sexes (57 [18.5\%] males and 32 [15.0\%] females; $\chi^{2}=1.113, \mathrm{p}=0.291$ ). However, there were significant differences in the rates of diagnosis between racial subgroups $(55$ [16.5\%] Caucasians and 5 [6.2\%] African Americans; $\chi^{2}=5.44, \mathrm{p}=0.02$ ).

The CI was collected for patients in the CI cohort (n $=1429$, although data were missing for each variable) to obtain brachycephaly measurements. Total cohort differences in mean CI based on sex, race, and ethnicity are summarized in Table 3. For the entire cohort with available data, the mean CI values were not significantly different when stratified by sex. However, when the mean CI values were compared across races and ethnicity, significance was achieved $(\mathrm{p}<0.001)$. The mean CI differences were also assessed directly between Caucasian and African American patients and were significant $(\mathrm{p}<0.001)$.

A subset analysis was conducted for children with available data who were within the treatment window of less than 24 months for CI measurements. Comparison of the CI for the subset analysis based on sex, race, and ethnicity is shown in Table 4. The differences in mean CI measurements for the treatment window based on sex were not significant. However, significance was achieved when stratifying mean CI based on race and ethnicity ( $p$ $<0.001)$.

\section{Discussion}

Positional cranial deformities have increased in the past few decades. ${ }^{4,5,7,11}$ Most prominently, since the introduction of the Back to Sleep campaign in 1992, a decrease in SIDS-related deaths was seen with a concomitant increase in PPP, ${ }^{710,12,13}$ While prominent risk factors for these conditions have been assessed, especially obstetric 
TABLE 2. Plagiocephaly demographic information for subset group in treatment window (2-24 months of age)

\begin{tabular}{lccc}
\hline \multicolumn{1}{c}{ Subset Cohort } & $\begin{array}{c}\text { No. of Patients } \\
(\%)\end{array}$ & Mean CVAI & p Value \\
\hline Total & 520 & 3.235 & \\
\hline Sex $(\mathrm{n}=520)$ & & & 0.404 \\
$\quad$ Male & $307(59 \%)$ & 3.397 & \\
$\quad$ Female & $213(41 \%)$ & 3.184 & \\
\hline Race $(\mathrm{n}=421)$ & & & $<0.0 \mathbf{0 1}^{*}$ \\
Caucasian & $334(79.3 \%)$ & 3.520 & \\
African American & $80(19.0 \%)$ & 2.750 & \\
Asian/Indian & $5(0.9 \%)$ & 7.134 & \\
American Indian & $2(0.5 \%)$ & 4.571 & \\
\hline Ethnicity $(\mathrm{n}=483)$ & & & 0.600 \\
$\quad$ Hispanic & $47(9.7 \%)$ & 3.419 & \\
$\quad$ Non-Hispanic/Latino & $436(90.3 \%)$ & 3.257 & \\
\hline
\end{tabular}

Boldface type indicates statistical significance.

* Independent t-test between Caucasian and African American subgroups.

and infant care factors, there have been no formal evaluations of demographic associations across sex, race, and ethnicity. ${ }^{14}$ For this reason, we set out to investigate the relationships therein. Our hypothesis was that cultural and/ or anthropological differences between sex, racial, and ethnic subgroups may contribute to differences in cranial deformation.

While the CVAI measurement was noted to be significantly different between males and females of all ages $(0-$ 19 years), there were no significant differences within the clinical treatment window. Similarly, there were no differences in rates of PPP diagnosis in males and females overall across either cohort. In terms of brachycephaly, there were no significant differences in CI between sexes within either age cohort. These findings suggest, that, although there may be statistically different values for CVAI measurements in older children, consistent with the literature, there is no clinically significant association between sex and cranial deformation. ${ }^{7,13}$

With regard to race, the CVAI measurement was significantly different between African American and Caucasian patients within the 2 cohorts. Additionally, PPP diagnosis rates were significantly higher among the Caucasian population in both cohorts. Concerning symmetric skull measurements, the CI was also found to be significantly higher in the Caucasian subgroups for both age cohorts and consistently above the brachycephaly threshold. Although the CI was higher in Caucasians, it may not represent a significant clinical or cosmetic burden on average. These findings represent a consistent association between Caucasian race and prevalence of cranial deformations. This substantial trend was consistent across both asymmetric and symmetric cranial deformation differences between racial subgroups. Therefore, Caucasians may clinically represent the greater burden of the plagiocephaly and brachycephaly populations.

Analysis of patients of Hispanic/Latin American descent was less consistent in terms of associations with symmetric and asymmetric deformation. While no significant results were discovered through analysis of asymmetrical
TABLE 3. Brachycephaly demographic information for total cohort

\begin{tabular}{lccc}
\hline \multicolumn{1}{c}{ Total Cohort } & No. of Patients (\%) & Mean Cl & p Value \\
\hline Total & 1499 & 82.517 & \\
\hline Sex $(\mathrm{n}=1494)$ & & & 0.450 \\
$\quad$ Male & $849(56.8)$ & 82.643 & \\
$\quad$ Female & $645(43.2)$ & 82.351 & \\
\hline Race $(\mathrm{n}=1286)$ & & & $<0.001^{*}$ \\
$\quad$ Caucasian & $1007(78.3)$ & 82.623 & \\
African American & $248(19.3)$ & 79.958 & \\
Asian/Indian & $26(2.0)$ & 82.398 & \\
$\quad$ American Indian & $5(0.4)$ & 81.328 & \\
\hline Ethnicity ( $\mathrm{n}=1320)$ & & & $<0.001$ \\
$\quad$ Hispanic & $138(10.5)$ & 84.745 & \\
Non-Hispanic/Latino & $1182(89.5)$ & 82.003 &
\end{tabular}

Boldface type indicates statistical significance.

* Independent t-test between Caucasian and African American subgroups.

deformations vis-à-vis plagiocephaly, symmetric deformations showed consistent differences in both cohorts for cranial index. The differences found between Hispanic/ Latin American and non-Hispanic/Latin American ethnicities would suggest a potential clinical association for brachycephaly; however, again the degree of clinical and cosmetic impact may be minimal on average.

While these differences appear novel at first glance, there are potential explanations in a variety of anthropological, socioeconomic, and cultural mechanisms. Since the 1960s, anthropological measurements of cranial "discriminant functions" have been used with a specificity between $82 \%$ and $89 \%$ to identify the race and sex of skeletal remains. ${ }^{5,15-17}$ Some of these discriminant functions identified included more rectangular orbits in African American subgroups, which could account for a more symmetric CVAI measurement. Similarly, the African American subgroups were known to have a more dolichocephalic skull, which would affect brachycephaly measurements. ${ }^{15}$ These

TABLE 4. Brachycephaly demographic information for subset group in treatment window (2-24 months of age)

\begin{tabular}{lccc}
\hline \multicolumn{1}{c}{ Subset Cohort } & Population (\%) & Mean Cl & p Value \\
\hline Total & 630 & 85.459 & \\
\hline Sex $(\mathrm{n}=630)$ & & & 0.534 \\
$\quad$ Male & $373(59.2)$ & 85.611 & \\
$\quad$ Female & $257(40.8)$ & 85.237 & \\
\hline Race $(\mathrm{n}=492)$ & & & $<0.001^{*}$ \\
$\quad$ Caucasian & $403(81.9)$ & 82.623 & \\
African American & $84(17.1)$ & 79.958 & \\
Asian/Indian & $3(0.6)$ & 82.398 & \\
American Indian & $2(0.4)$ & 81.328 & \\
\hline Ethnicity ( $\mathrm{n}=528)$ & & & $<0.001$ \\
Hispanic & $55(10.4)$ & 87.890 & \\
Non-Hispanic/Latino & $473(89.6)$ & 84.972 & \\
\hline
\end{tabular}

Boldface type indicates statistical significance.

* Independent t-test between Caucasian and African American subgroups. 
noted differences in combination with any number of other cranial bone variations could account for some degree of variation in cranial deformation seen between races.

Historically, studies have shown that African American mothers and mothers in low socioeconomic communities have been less likely to comply with the recommendations of the Back to Sleep campaign. ${ }^{18-21}$ The children show consistently higher rates of prone sleeping, even though studies have shown that the mothers are generally aware of the campaign. ${ }^{19}$ The reasons for these findings have focused on the influence of matriarchal figures such as grandmothers, lack of faith in the supine position being "safer," and not enough trust in their pediatrician. ${ }^{18-21}$ Given the effect of the Back to Sleep campaign on positional cranial deformations, specifically PPP, these findings would also be consistent with fewer cranial deformations in infants and children of African American and Hispanic/Latin American mothers as well as those born into low socioeconomic status communities.

Some notable limitations of this study are that we did not evaluate any patients longitudinally, so we were unable to assess if any patients had previously been treated and what impact that may have on these cohorts. Second, the radiographic evidence provided in this investigation was not verified by clinical assessment of these measurements via craniometry and therefore may be subject to variations within the differences of those measurement scales. Lastly, sex, race, and ethnicity had patients with missing data or "other" responses for both CVAI and CI measurements.

\section{Conclusions}

Clinically, cranial deformation manifested in the forms of plagiocephaly and brachycephaly is more likely to be seen in Caucasian populations than in African American populations. Explanations for these variations may be due to anthropomorphic differences in cranial anatomy as well as cultural differences in infant sleeping positions.

\section{References}

1. Feijen M, Franssen B, Vincken N, van der Hulst RR. Prevalence and consequences of positional plagiocephaly and brachycephaly. J Craniofac Surg. 2015;26(8):e770-e773.

2. Linz C, Kunz F, Böhm H, Schweitzer T. Positional skull deformities. Dtsch Arztebl Int. 2017;114(31-32):535-542.

3. Dwyer T, Ponsonby AL. Sudden infant death syndrome and prone sleeping position. Ann Epidemiol. 2009;19(4):245-249.

4. Moon RY. SIDS and other sleep-related infant deaths: expansion of recommendations for a safe infant sleeping environment. Pediatrics. 2011;128(5):e1341-e1367.

5. Turk AE, McCarthy JG, Thorne CH, Wisoff JH. The "back to sleep campaign" and deformational plagiocephaly: is there cause for concern? J Craniofac Surg. 1996;7(1):12-18.

6. Kelly KM, Joganic EF, Beals SP, et al. Helmet treatment of infants with deformational brachycephaly. Glob Pediatr Health. 2018;5:2333794x18805618.

7. Di Rocco F, Ble V, Beuriat PA, et al. Prevalence and severity of positional plagiocephaly in children and adolescents. Acta Neurochir (Wien). 2019;161(6):1095-1098.

8. Likus W, Bajor G, Gruszczyńska K, et al. Cephalic index in the first three years of life: study of children with normal brain development based on computed tomography. ScientificWorldJournal. 2014;2014:502836.

9. Koizumi T, Komuro Y, Hashizume K, Yanai A. Cephalic index of Japanese children with normal brain development. $J$ Craniofac Surg. 2010;21(5):1434-1437.

10. Graham JM Jr, Kreutzman J, Earl D, et al. Deformational brachycephaly in supine-sleeping infants. $J$ Pediatr. 2005;146(2):253-257.

11. Biggs WS. Diagnosis and management of positional head deformity. Am Fam Physician. 2003;67(9):1953-1956.

12. Hutchison BL, Hutchison LA, Thompson JM, Mitchell EA. Plagiocephaly and brachycephaly in the first two years of life: a prospective cohort study. Pediatrics. 2004;114(4):970-980.

13. Ahluwalia R, Kiely C, Foster J, et al. Positional posterior plagiocephaly: a single-center review. J Neurosurg Pediatr. 2020:25(5):514-518.

14. Bialocerkowski AE, Vladusic SL, Wei Ng C. Prevalence, risk factors, and natural history of positional plagiocephaly: a systematic review. Dev Med Child Neurol. 2008;50(8):577-586.

15. Eugene Giles EO. Sex determination by discriminant function analysis of crania. Am J Phys Anthropol. 1963;21:53-68.

16. Nikita E, Michopoulou E. A quantitative approach for sex estimation based on cranial morphology. Am J Phys Anthropol. 2018;165(3):507-517.

17. Church MS. Determination of race from the skeleton through forensic anthropological methods. Forensic Sci Rev. 1995;7(1):1-39.

18. Colson ER, Levenson S, Rybin D, et al. Barriers to following the supine sleep recommendation among mothers at four centers for the Women, Infants, and Children Program. Pediatrics. 2006;118(2):e243-e250.

19. Mathews AA, Joyner BL, Oden RP, et al. Comparison of infant sleep practices in African-American and US Hispanic families: implications for sleep-related infant death. J Immigr Minor Health. 2015;17(3):834-842.

20. Oden RP, Joyner BL, Ajao TI, Moon RY. Factors influencing African American mothers' decisions about sleep position: a qualitative study. J Natl Med Assoc. 2010;102(10):870-872, 875-880.

21. Brenner RA, Simons-Morton BG, Bhaskar B, et al. Prevalence and predictors of the prone sleep position among innercity infants. JAMA. 1998;280(4):341-346.

\section{Disclosures}

The authors report no conflict of interest concerning the materials or methods used in this study or the findings specified in this paper.

\section{Author Contributions}

Conception and design: Foster. Acquisition of data: Foster, Ahluwalia, Sherburn, Kelly, Kiely. Analysis and interpretation of data: Foster, Ahluwalia, Kelly, Sellyn. Drafting the article: Foster, Ahluwalia, Sherburn, Kelly, Sellyn, Bonfield. Critically revising the article: Foster, Ahluwalia, Shannon, Bonfield. Reviewed submitted version of manuscript: Foster, Ahluwalia, Shannon, Bonfield. Approved the final version of the manuscript on behalf of all authors: Foster. Statistical analysis: Foster. Administrative/ technical/material support: Wiseman, Gannon, Shannon, Bonfield. Study supervision: Wiseman, Bonfield.

\section{Supplemental Information}

\section{Previous Presentations}

Portions of this paper were presented in abstract form at the 71st Southern Neurosurgical Society Annual Meeting, Scottsdale, Arizona, February 26-29, 2020.

\section{Correspondence}

Jarrett Foster: Surgical Outcomes Center for Kids (SOCKs), Nashville, TN. jarrett.foster@uscmed.sc.edu. 